\title{
Modelos de distribución potencial de mamíferos nativos en la Patagonia
}

\author{
Melina E. Zuliani ${ }^{\bowtie}$ \&. Adrián Monjeau \\ Departamento de Análisis de Sistemas Complejos. Fundación Bariloche-CONICET.
}

\begin{abstract}
Resumen. Ante las previsiones sobre el cambio climático y su influencia en los rangos geográficos de las especies, es importante esclarecer qué indicadores climáticos son los que mejor explican las distribuciones actuales, como herramientas de conservación de especies amenazadas. Asimismo, es relevante conocer el porcentaje de protección que tienen los hábitats idóneos para dichas especies. En este trabajo identificamos las principales variables climáticas que explican los patrones de distribución de 16 especies de mamíferos medianos y grandes en la Patagonia mediante el modelo MaxEnt, utilizando 19 variables bioclimáticas del WorldClim, la elevación, el NDVI, el EVI y un proxy de presión antrópica (human footprint). Calculamos el porcentaje de cobertura de áreas protegidas para cada especie y encontramos que la mayor parte del hábitat ideal está fuera de ellas, y que las áreas de protección estricta (I y II) tienen más registros de presencia que las áreas de recursos manejados (III a VI). Las especies de distribución restringida son las más vulnerables a la extinción por ser menos resilientes a los cambios en la envoltura climática, sobre todo fuera de áreas protegidas.
\end{abstract}

[Palabras clave: envoltura climática, modelo de distribución, Patagonia, mamíferos nativos]

\begin{abstract}
Potential distribution models of native mammals in Patagonia. Given the prospects of climate change and its influence on the species' geographic range, it is important to clarify which climate indicators best explain current distributions, as tools for the conservation of threatened species. It is also important to know the percentage of protection of suitable habitats for these species. In this paper we identify the main climatic variables that explain the distribution patterns of 16 species of medium and large mammals in Patagonia through the MaxEnt model, using 19 bioclimatic variables from WorldClim, elevation, NDVI and EVI and a proxy for anthropic pressure (human footprint). We calculated the percentage of coverage of protected areas for each species and found that most of the ideal habitat is outside them, and that strictly protected areas (I and II) have more records of presence than managed resource areas (III to VI). Species with restricted distributions are the most vulnerable to extinction because they are less resilient to changes in the climatic envelope, especially outside protected areas.
\end{abstract}

[Keywords: climate envelope, distribution model, Patagonia, native mammals]

\section{INTRODUCCIÓN}

Existe una controversia científica de más de 200 años al tratar de entender las causas de la extinción o la supervivencia de las especies. De manera esquemática, la colección de explicaciones puede aglomerarse en tres grandes grupos: causas biológicas, ambientales y antrópicas (Abramson et al. 2017; Monjeau et al. 2017). La historia evolutiva de los taxones, tanto con sus linajes extintos como con los que han prevalecido hasta el presente es una combinación de estos tres factores, con distintos grados de influencia según el lugar donde ocurrieron. Comprender las causas de la presencia o la ausencia de las especies, o de extinción o supervivencia en un sitio dado es esencial para tomar medidas de conservación efectiva.
Un síntoma espacialmente explícito de esta complejidad multicausal es el rango geográfico de las especies; el tamaño y el área de distribución es una compleja expresión de su ecología y su historia evolutiva (Brown 1995). Esta área está moldeada por múltiples factores (e.g., bióticos, abióticos e interacciones con los humanos) que actúan de manera dinámica con diferentes fortalezas y en distintas escalas (Pulliam 2000). La interacción con estos factores moldea la forma y el tamaño del área de distribución de las especies (Rapoport 1982; Soberón and Pearson 2005). De los factores mencionados, el clima es el que ejerce mayor influencia en los límites de distribución, cuyos patrones son detectables a escala regional o continental (Pearson and Dawson 2003). En los últimos años, el modelado de nicho (ENM 
por sus siglas en inglés) ha sido muy utilizado para delimitar la distribución geográfica de las especies. Sus resultados son estadísticamente robustos (Peterson et al. 2011) y han sido usados para fines muy variados: entre ellos, la conservación (Lim et al. 2002; Peterson et al. 2002; Guisan and Thuiller 2005; Johnson and Gillingham 2005; Ortega-Huerta and Peterson 2005; Miller 2010). Dentro de los análisis que se pueden realizar con el ENM está la distribución potencial, que es una proyección geográfica que combina variables ambientales, biológicas y antrópicas asociadas a puntos de presencia de los organismos (Soberón and Pearson 2005; Brown 1995; Kearney and Porter 2004). Esta última es la que utilizamos en la presente investigación.

La Patagonia argentina presenta un amplio territorio con una distribución de paisajes muy influenciada por la cordillera de los Andes, que desde su ascenso generó un gradiente ambiental de oeste a este. Esta zona presenta una composición de fauna y flora muy particular, adaptada a los climas extremos de la región. Hace casi 100 años, cuando los colonos comenzaron a poblar esta zona, este paisaje cambió notablemente; ahora es una zona más productiva que presenta un cambio en la fisionomía vegetal y, por lo tanto, también en la composición de la fauna. En la actualidad, la mayoría de las especies están expuestas a factores como la pérdida, la degradación y la fragmentación del hábitat, la caza furtiva, la interferencia y la competencia con especies exóticas, la depredación por perros, las forestaciones con plantas exóticas, las enfermedades transmitidas por el ganado, etc. Todo esto provoca el retroceso en la distribución y la disminución de las poblacionales (Corti et al. 2010; Jiménez 2010; Napolitano et al. 2012; Lucherini et al. 2019). La primera área protegida de la Argentina, el Parque Nacional del Sur, se ubicó en la Patagonia; su objetivo era preservar las bellezas escénicas y paisajísticas. Hoy, la región posee al menos 71 áreas protegidas que abarcan menos del $10 \%$ de su superficie. Estas áreas continúan persiguiendo el objetivo original y agregan, además, otros aspectos como los ambientales, culturales, científicos, educativos y sociales, lo que favorece su planificación y su manejo.

Este trabajo tiene como objetivos 1) delimitar la distribución potencial de quince especies de mamíferos medianos y grandes que habitan la Patagonia, utilizando indicadores climáticos, proxies de topografía, de vegetación y de presión antrópica, 2) estimar el porcentaje de la distribución potencial protegida, debido a que se encuentran dentro de las áreas protegidas, y 3) proponer recomendaciones para la conservación de las especies más amenazadas.

\section{Materiales y Métodos}

\section{Sitio y especies de estudio}

Nuestra área de estudio es la Patagonia, en la interpretación sensu lato del término según Monjeau et al. (1998). Sumado a la variación latitudinal entre los $32^{\circ} \mathrm{S}$ y los $55^{\circ} \mathrm{S}$, presenta, además, una elevación que varía desde 3000 $\mathrm{m}$ hasta el nivel del mar, con varias áreas de altitud intermedia a alta (e.g., norte y mesetas patagónicas australes como Somuncura, a $\sim 2000 \mathrm{~m}$ ), produciendo algunas de las transiciones bióticas más intensas conocidas (Veblen and Lorenz 1988). La elevación genera gradientes térmicos que influyen en los límites de distribución de las especies. La cordillera de los Andes es una barrera para los vientos húmedos formados en el Anticiclón del Pacífico Sur, que al elevarse se enfrían y descargan lluvias en el sector oeste de la Patagonia, y al descender hacia el este se calientan y captan la humedad del suelo. Este fenómeno genera un gradiente abrupto de precipitaciones, desde $2000 \mathrm{~mm}$ en la frontera con Chile hasta $200 \mathrm{~mm}$ en la costa atlántica, concentrándose en invierno y con déficit hídrico en el verano (Prohaska 1976; Paruelo et al. 1998). Asimismo, las zonas elevadas, al retener agua en forma de hielo y nieve o en lagunas de altura, son la fuente de agua permanente de toda la región, lo que también influye en el gradiente ambiental. Debido a estos factores y a su posición latitudinal, es una región templado-fría, con una temperatura media anual que varía entre $12^{\circ} \mathrm{C}$ en la parte noreste y $3^{\circ} \mathrm{C}$ hacia el sur.

Trabajamos con 15 especies de mamíferos nativos medianos (1-15 kg) y grandes (>15 $\mathrm{kg}$ ): Chaetophractus villosus (peludo), Conepatus chinga (zorrino), Dolichotis patagonum (mara), Galictis cuja (hurón), Hippocamelus bisulcus (huemul), Lama guanicoe (guanaco), Leopardus colocolo (gato del pajonal), Leopardus geoffroyi (gato móntes), Leopardus guigna (gato huiña), Lycalopex culpaeus (zorro colorado), Lycalopex gymnocercus (zorro gris), Lyncodon patagonicus (huroncito patágonico), Pudu puda (pudú), Puma concolor (puma) y Zaedyus pichiy (piche). 
Se consultó toda la bibliografía específica referida a mamíferos de la región patagónica con el fin de completar las localidades de registro de ocurrencia (i.e., coordenadas geográficas de latitud y longitud y fecha de registro) de cada una de las especies. Recopilamos un total de 716 registros, que luego de ser procesados con una cuadrícula de $10 \times 10 \mathrm{~km}$ resultó en 574 registros de presencia confirmada.

\section{Variables y modelos}

Datos ambientales. Se utilizaron 20 variables ambientales de la base de datos WorldClim (Lim et al. 2002; Fick and Hijmans 2017), incluida la elevación y 19 variables bioclimáticas con una resolución de 30 arco segundos, proyectadas en WGS 1984, siguiendo a Hijmans et al. 2005. Las variables ambientales que combinan temperatura y precipitación (e.g., Bio 8, 9, 18 y 19) fueron excluidas porque presentan extrañas anomalías espaciales y discontinuidades entre píxeles vecinos (Escobar et al. 2014; Astorga et al. 2018). Se sumaron otras dos variables ambientales representativas, el Índice de Vegetación de Diferencia Normalizada (NDVI) y el Î́ndice de Vegetación Mejorado (EVI), con el fin de contrastar su poder predictivo con las variables climáticas. Estos dos índices se obtuvieron procesando una serie de imágenes satelitales MODIS. Además, se enmascararon áreas 'descartando lo improbable', que consiste en recortar del área de estudio a lagos y ríos donde nuestras especies terrestres no habitan. Añadimos el índice de huella humana (HFP) (Sanderson et al. 2002) como variable para controlar el sesgo del modelo.

Para validar cada uno de nuestros modelos realizamos una validación cruzada y calculamos el área bajo la curva (AUC), que es una característica operativa del receptor (ROC) (Kuemmerle et al. 2011). Realizamos un análisis exploratorio con MaxEnt 3.3.3k (Phillips et al. 2006) con las variables seleccionadas. Luego realizamos un análisis de correlación de Pearson, en el que una correlación de 0.8 se consideró alta (Dormann et al. 2012) y nos llevó a descartar todas las variables altamente correlacionadas. Dentro de cada grupo de variables no correlacionadas utilizamos la prueba Jackknife para detectar cuáles variables mostraban el mayor aporte explicativo, considerando también aquellas que, según criterio de expertos, tienen significación biológica para cada una de las especies estudiadas (Núñez Penichet et al. 2016), y descartando las demás de la búsqueda de causas.

Elegimos el algoritmo de máxima entropía (MaxEnt) porque genera resultados óptimos utilizando sólo puntos de presencia, como es nuestro caso (Elith et al. 2006; Peterson et al. 2007). Además, se desempeña bien con tamaños de muestra pequeños (Wisz et al. 2008), incorpora efectos de interacción de variables ambientales y es un algoritmo determinista, lo que significa que los resultados siempre convergen en una distribución de probabilidad óptima única (Phillips et al. 2006). Los modelos MaxEnt se generaron para cada especie utilizando un $25 \%$ de datos de prueba, corridas aleatorias sin correlación alguna, 1000 iteraciones, 10 repeticiones, 10000 puntos de fondo y la opción acumulativa como formato de salida (Phillips and Dudik 2008; Merow et al. 2013; Ruiz Barlett et al. 2019).

Definición del área de modelado o área $\mathrm{M}$. $\mathrm{El}$ área $\mathrm{M}$ es la región que tuvo disponible una especie para su uso desde los orígenes de dicha especie, independientemente de si fue utilizada, o bien alguna región definida en un momento temporal relevante Svenning and Skov 2004). Esta área proporciona un conjunto similar de variables ambientales que podría influir en la distribución de las especies (Soberón and Peterson 2005; Barve et al. 2011). El área M tiene efectos importantes en los resultados de los ejercicios de modelado de nichos ecológicos debido a que se toman muestras de predictores para los puntos de fondo (Barve et al. 2011). Para las especies que presentan una distribución reducida ( $P$. puda, H. bisulcus, L. guigna, L. colocolo y D. patagonum) utilizamos su área de distribución histórica. Para las especies que presentan una amplia distribución (L. geoffroyi, P. concolor, G. cuja, L. patagonicus, C. chinga, L. gymnocercus, L. culpaeus, C. villosus, Z. pichiy y L. guanicoe) escogimos la Patagonia como área de estudio, que para este propósito definimos como el extremo sur de América del Sur, entre $32^{\circ} \mathrm{S}$ y $52^{\circ} \mathrm{S}$. El área de estudio fue seleccionada porque abarca una fracción de la distribución actual e histórica de estas especies.

Procedimiento de modelado y evaluación. Para el ajuste del modelo usamos la función ENMevaluate del paquete de R ENMeval (Muscarella et al. 2014), utilizando las funciones lineal $(\mathrm{L})$, cuadrático $(\mathrm{Q})$, producto $(\mathrm{P})$, bisagra $(\mathrm{H})$ y umbral $(\mathrm{T})$, según el 
número de observaciones. Para especies con '80 registros de ocurrencia usamos $\mathrm{L}$, LQ y LQP; para especies con más de 80 registros utilizamos $\mathrm{L}, \mathrm{LQ}, \mathrm{LQP}, \mathrm{H}, \mathrm{LQH}$, LQHP y LQHPT (Merow et al. 2013). Estas características se combinaron con diferentes multiplicadores de regularización (rm): 0.5 a 5 en pasos crecientes de 0.5. Para todas las especies se utilizó un método de validación cruzada de particiones de tipo bloque (González et al. 2021). Generamos modelos preliminares para cada una de las especies usando tres multiplicadores de regularización (i.e., $§=0.5,1$ y 2 ), ya que se sabe que estos valores generan resultados diferentes (Warren and Seifert 2011; Radosavljevic and Anderson 2014), y se utilizó el área bajo la curva (AUC) como un estimador del poder predictivo de cada modelo, utilizando las características operativas del receptor (Phillips et al. 2004, 2006). Dentro de los modelos candidatos se seleccionaron según el valor mínimo para el AICc y promediados de acuerdo con sus correspondientes ponderaciones de Akaike (Bouchet and Meeuwig 2015). Los mapas de la especie se generaron utilizando los valores medios proporcionados por MaxEnt (Martin 2010; Schiaffini et al. 2013; Ruiz Barlett et al. 2019). Elegimos los valores de la mediana porque representan una caracterización estadística más robusta que la media, en particular para la función de densidad de probabilidad de una población biológica, que tiene naturalmente un límite inferior igual a cero (Feller 1967). Los detalles de los pasos para la construcción y análisis de los modelos de MaxEnt se describen a través del protocolo ODMAP (descripción general, datos, modelo, evaluación y predicción) siguiendo a Zurrel et al. (2020; Tabla S1 del Material Suplementario). Los mapas de cada especie se evaluaron mediante un examen visual cualitativo, basado en nuestra experiencia de campo, mapas de distribución de especies (Vila et al. 2010; Díaz et al. 2013; Jiménez 2010; Vale et al. 2015; Schiaffini 2014) y tipos de hábitats donde se sabe que se encuentra la especie (León et al. 1998; Paruelo et al. 1998).

Análisis de conservación. Un área natural protegida "está consagrada a la protección y mantenimiento de la diversidad biológica, de los recursos naturales y culturales asociados, y manejada a través de medios jurídicos eficaces" (IUCN 2012). Existen dos grandes tipos de categorías de áreas protegidas: las IUCN- I y II (APCIyII) de conservación estricta y las áreas protegidas de categorías IUCN- III a VI
(APCIIIaVI) de recursos manejados. A cada uno de los modelos generados se le superpuso la capa de áreas protegidas de la Patagonia y se calculó el porcentaje de área de distribución potencial que coincide con las áreas protegidas categorías APCIyII y APCIIIaVI. Los sistemas de áreas protegidas combinan generalmente un mosaico de distintas categorías, lo que determina una gobernanza compleja, interactiva y participativa (Monjeau 2010). Finalmente se generó un mapa donde se muestra la zona con mayor riqueza de especies que se encuentran dentro de la categoría "en peligro de extinción" (cuando el taxón enfrenta un alto riesgo de extinción o deterioro poblacional en estado silvestre en el futuro cercano, según queda definido por cualquiera de los criterios $\mathrm{A}$ a $\mathrm{E}$, IUCN 2017) y "vulnerable" (la mejor evidencia disponible indica que este taxón enfrenta un moderado riesgo de extinción o deterioro poblacional a mediano plazo, según lo definido por los criterios A a E, IUCN 2017), sobre la base de la superposición de la distribución potencial, el cual se generó al reclasificar las imágenes raster de cada especie con la superposición de las áreas protegidas nacionales y provinciales.

\section{Resultados}

\section{Escenarios predictores}

Se obtuvieron valores bajos para los AUC train y AUC promedio para los diferentes escenarios predictores, los cuales son mayores con valores reducidos de rm (0.5). La métrica AICc se comportó de manera dispar para los diferentes valores de $\mathrm{rm}$, pero a medida que éstos aumentan, los modelos mejoran sus rendimientos para algunas especies (Tabla 1).

\section{Resultados del modelo}

Para la mayoría de las especies, los modelos de nicho mostraron un buen poder predictivo, con valores de AUCTest entre $0.800 \pm 0.090$ y $0.931 \pm 0.090$. Sin embargo, para el resto de las especies, el valor predictivo fue menor a 0.8 (Tabla 2 y Tabla 2 bis).

Se generaron modelos de distribución potencial para las 15 especies en estudio distribuidas por la Patagonia (Figuras 1, 2 y 3). Los registros de ocurrencia marginal varían dependiendo de la especie, ya que algunos de estos exhiben una amplia distribución y están presentes en casi toda la Patagonia (Leopardus 
Tabla 1. Métricas de desempeño de modelos MaxEnt para especies de mamíferos nativos de la Patagonia. Las métricas presentadas corresponden a los modelos elegidos después de filtrar los resultados y de la inspección visual.

Table 1. Performance metrics of MaxEnt models for native mammal species of Patagonia. The metrics correspond to the chosen models after filtering results and visual inspection.

\begin{tabular}{lccccccc}
\hline Especie & $\begin{array}{c}\text { Validación } \\
\text { cruzada }\end{array}$ & $\begin{array}{c}\text { Caracte- } \\
\text { rísticas }\end{array}$ & rm & $\begin{array}{c}\text { Train } \\
\text { AUC }\end{array}$ & $\begin{array}{c}\text { Pruebas promedio } \\
\text { AUC }\end{array}$ & $\begin{array}{c}\text { Pruebas promedio } \\
\text { MTP }\end{array}$ & AICc \\
\hline Hippocamelus bisulcus & block & LQ & 5.5 & 0.59 & 0.56 & 0.019 & 1599.275 \\
Pudu puda & block & LQ & 0.5 & 0.59 & 0.11 & 0.096 & 1320.41 \\
Leopardus guigna & block & L & 0.5 & 0.75 & 0.74 & 0.083 & 332.94 \\
Dolichotis patagonum & block & LQP & 2 & 0.5 & 0.5 & 0.041 & 582.79 \\
Leopardus geoffroyi & block & L & 2 & 0.5 & 0.5 & 0 & 488.44 \\
Leopardus colocolo & block & L & 2 & 0.5 & 0.47 & 0 & 464.39 \\
Puma concolor & block & Q & 3 & 0.56 & 0.5 & 0.01 & 2553.46 \\
Conepatus chinga & block & LQP & 1.5 & 0.68 & 0.64 & 0.05 & 578.85 \\
Lycalopex gymnocercus & block & LQP & 0.5 & 0.73 & 0.65 & 0.04 & 1676.36 \\
Lycalopex culpaeus & block & LQP & 4.5 & 0.5 & 0.5 & 0 & 969.78 \\
Galictis cuja & block & LQP & 3.5 & 0.55 & 0.48 & 0 & 779.5 \\
Lyncodon patagonicus & block & LQP & 2 & 0.67 & 0.52 & 0.11 & 891.55 \\
Chaetophractus villosus & block & LQP & 3 & 0.5 & 0.5 & 0.18 & 671.84 \\
Zaedyus pichiy & block & LQP & 0.5 & 0.74 & 0.61 & 0.15 & 576.77 \\
Lama guanicoe & block & LQP & 3.5 & 0.5 & 0.49 & 0.49 & 1578.23 \\
\hline
\end{tabular}

Tabla 2. Estimaciones heurísticas de la contribución relativa de la variable ambiental (en porcentaje) de los modelos generados para cada especie. Porcentaje variable (\%) y valores de permutación (e.g., información que no está presente en el resto de las variables, P) se muestran para siete especies. Las variables con las puntuaciones más altas para \% y $\mathrm{P}$ se indican en negrita. Entre paréntesis, para cada especie figura el modelo usado, sobre la base de su parámetro de regularización $\beta$ (e.g., $0.5,1,2$ ) para mapear y describir las variables ambientales claves.

Table 2. Heuristic estimates of relative environmental variable contribution (in percentage) for the models generated for each species. Variable percentage (\%) and permutation values (i.e., information not present in the rest of the variables, $\mathrm{P})$ are presented for seven species. Variables with the highest scores for $\%$ and $\mathrm{P}$ are indicated in bold. In parenthesis for each species, the model used is shown, based on its regularization parameter $\beta$ (i.e., $0.5,1,2$ ), to map and describe the key environmental variables.

\begin{tabular}{|c|c|c|c|c|c|c|c|}
\hline$\overline{\text { Variable }}$ & $\begin{array}{l}\text { Pudu puda } \\
\text { (0.5) }\end{array}$ & $\begin{array}{c}\text { Hippocamelus } \\
\text { bisulcus } \\
(0.5)\end{array}$ & $\begin{array}{c}\text { Leopardus } \\
\text { guigna } \\
(0.5)\end{array}$ & $\begin{array}{c}\text { Leopardus } \\
\text { geoffroyi } \\
\text { (1) }\end{array}$ & $\begin{array}{l}\text { Leopardus } \\
\text { colocolo } \\
\text { (2) }\end{array}$ & $\begin{array}{c}\text { Puma } \\
\text { concolor } \\
(0.5)\end{array}$ & $\begin{array}{l}\text { Galictis } \\
\text { cuja } \\
(0.5)\end{array}$ \\
\hline Temperatura media anual & & & & & & & $28.5-4.5$ \\
\hline Rango de temperatura diurno medio & & $2.8-0.4$ & $0.1-0$ & $0.3-0$ & & & \\
\hline Isotermalidad & $18-18.1$ & & & & $57.2-9.8$ & $4.3-5.8$ & \\
\hline Estacionalidad de temperatura & & $4.5-11.5$ & $0.3-0.5$ & $18.9-39.3$ & & $8.1-17.3$ & $8.8-1.7$ \\
\hline Temperatura máxima del mes más cálido & $33.6-22.1$ & & & & & & \\
\hline Temperatura mínima del mes más frío & $1.9-2.7$ & $17.2-11.8$ & $54.4-70.9$ & & & $4.5-12$ & $3.6-0.3$ \\
\hline Rango de temperatura anual & & & & & & $16.1-10.9$ & \\
\hline Temperatura media del trimestre más frío & & $0.4-0$ & & $0.8-8$ & 29.4-0-1 & & $2-14.8$ \\
\hline $\begin{array}{l}\text { Temperatura media del trimestre más } \\
\text { cálido }\end{array}$ & $0.7-11$ & $1.5-1.3$ & $1-3.5$ & & & & \\
\hline Precipitación total anual & & 7.5-19.1 & & & $6.2-0-2$ & $31.6-16.9$ & $1.8-8.2$ \\
\hline Precipitación del mes más húmedo & $1.4-8.1$ & & $27.5-28.2$ & & & & \\
\hline Precipitación del mes más seco & 14.5-31.6 & & & $63-30.9$ & & $2.7-21.7$ & $5.3-31.4$ \\
\hline Estacionalidad de la precipitación & $22.8-0.3$ & & & & $0.4-4.2$ & & $1.2-1.9$ \\
\hline Precipitación del trimestre más húmedo & & $19.9-32.7$ & 2.1-0 & $11.4-9.2$ & & & \\
\hline Precipitación del trimestre más seco & & & & & & 33.4-3.7 & \\
\hline Altitud & 2.9-0.1 & $4.7-3.2$ & $9.2-10.1$ & $1.7-2.6$ & $0.4-4.2$ & $1.4-11.6$ & $19.7-7.3$ \\
\hline Índice de Vegetación Mejorado & $0.1-0.1$ & $1.2-4.4$ & & & & & $1.7-4$ \\
\hline $\begin{array}{l}\text { Índice de Vegetación de Diferencia } \\
\text { Normalizada }\end{array}$ & & $0.6-3.7$ & $3.9-15.9$ & $44.2-36.3$ & & $7.8-3$ & $0.6-1.2$ \\
\hline Índice de Huella Humana & $1.8-0.2$ & $39.9-12$ & $1.5-3.6$ & $50.6-61.8$ & & 6-8 & 29.4-29.6 \\
\hline Total & 100 & 100 & 100 & 100 & 100 & 100 & 99.9 \\
\hline $\begin{array}{l}\text { \% explicado por las dos variables más } \\
\text { importantes }\end{array}$ & 56.4 & 59.8 & 81.9 & 81.9 & 86.6 & 65 & 57.9 \\
\hline $\begin{array}{l}\% \text { explicado por variables superiores al } \\
10 \%(\mathrm{n})\end{array}$ & $88.9(4)$ & $77(3)$ & $81.9(2)$ & $93.3(3)$ & $86.6(2)$ & $65(2)$ & $77.6(3)$ \\
\hline AUCTest & $\begin{array}{c}0.889 \\
\pm 0.096\end{array}$ & $\begin{array}{c}0.764 \\
\pm 0.191\end{array}$ & $\begin{array}{c}0.889 \\
\pm 0.053\end{array}$ & $\begin{array}{c}0.755 \\
\pm 0.100\end{array}$ & $\begin{array}{c}0.684 \\
\pm 0.308\end{array}$ & $\begin{array}{c}0.855 \\
\pm 0.063\end{array}$ & $\begin{array}{c}0.800 \\
\pm 0.090\end{array}$ \\
\hline
\end{tabular}


Tabla 2 bis. Estimaciones heurísticas de la contribución relativa de la variable ambiental (en porcentaje) de los modelos generados para cada especie. Porcentaje variable (\%) y valores de permutación (i.e., información que no está presente en el resto de las variables, $\mathrm{P}$ ) se muestran para otras ocho especies. Las variables con las puntuaciones más altas para $\%$ y $\mathrm{P}$ se indican en negrita. Entre paréntesis para cada especie figura el modelo que usamos, basado en su parámetro de regularización $\beta$ (i.e., $0.5,1,2$ ), para mapear y describir las variables ambientales claves.

Table 2 bis. Heuristic estimates of relative environmental variable contribution (in percentage) for the models generated for each species. Variable percentage (\%) and permutation values (i.e., information not present in the rest of the variables, $\mathrm{P}$ ) are presented for other eight species. Variables with the highest scores for $\%$ and $\mathrm{P}$ are indicated in bold. In parenthesis, for each species, the model used is shown, based on its regularization parameter $\beta$ (i.e., $0.5,1$, 2), to map and describe the key environmental variables.

\begin{tabular}{|c|c|c|c|c|c|c|c|c|}
\hline Variable & $\begin{array}{l}\text { Lyncodon } \\
\text { patagonicus } \\
\text { (2) }\end{array}$ & $\begin{array}{c}\text { Conepatus } \\
\text { chinga } \\
(0.5)\end{array}$ & $\begin{array}{l}\text { Lycalopex } \\
\text { gymnocercus } \\
(2)\end{array}$ & $\begin{array}{l}\text { Lycalopex } \\
\text { culpaeus } \\
\quad(1)\end{array}$ & $\begin{array}{c}\text { Chaetophractus } \\
\text { villosus } \\
(0.5)\end{array}$ & $\begin{array}{l}\text { Zaedyus } \\
\text { pichiy } \\
\text { (2) }\end{array}$ & $\begin{array}{l}\text { Lama } \\
\text { guanicoe } \\
(2)\end{array}$ & $\begin{array}{l}\text { Dolichotis } \\
\text { patagonum } \\
\text { (1) }\end{array}$ \\
\hline Temperatura media anual & & & & & & $13-0.5$ & & \\
\hline $\begin{array}{l}\text { Rango de temperatura diurno } \\
\text { medio }\end{array}$ & & & $0.8-1.4$ & $12.8-13.2$ & & & $0.4-0$ & $30.1-0$ \\
\hline Isotermalidad & $0.5-0.1$ & & & 2.6-1 & & & $1.1-0.9$ & \\
\hline Estacionalidad de la temperatura & & $28.6-30.7$ & $42.5-14.3$ & 20.1-27.5 & $25.4-17.5$ & $37.5-54.9$ & $46.8-29.9$ & $1-0.1$ \\
\hline $\begin{array}{l}\text { Temperatura máxima del mes } \\
\text { más cálido }\end{array}$ & $22.4-49.5$ & $1.6-0.5$ & & $1.8-0$ & $1.3-1.2$ & & & $16.8-10.4$ \\
\hline $\begin{array}{l}\text { Temperatura mínima del mes } \\
\text { más frío }\end{array}$ & $14.3-5.2$ & & $10-6.7$ & 3.1-5.4 & & $7.4-0.2$ & $10.6-21.1$ & 1.1-7.4 \\
\hline Rango de temperatura anual & & $7.5-11.3$ & $11.9-39.2$ & & $1.3-1.2$ & & $1.9-23-7$ & \\
\hline \multicolumn{9}{|l|}{$\begin{array}{l}\text { Temperatura media del trimestre } \\
\text { más frío }\end{array}$} \\
\hline $\begin{array}{l}\text { Temperatura media del trimestre } \\
\text { más cálido }\end{array}$ & $0-0.1$ & & & $1-6.9$ & & & & \\
\hline Precipitación total anual & $1.6-1$ & & $12.5-3.2$ & $1.1-7.2$ & $4.1-5.1$ & $11-11.8$ & & $1.4-17.3$ \\
\hline $\begin{array}{l}\text { Precipitación del mes más } \\
\text { húmedo }\end{array}$ & & $1-6.3$ & & & 2.6-3.6 & & $0.9-0.9$ & $3.8-30.6$ \\
\hline Precipitación del mes más seco & $5.1-13.1$ & & $1.3-1.5$ & $17.1-16.2$ & & & $3.1-4.3$ & \\
\hline $\begin{array}{l}\text { Estacionalidad de la } \\
\text { precipitación }\end{array}$ & 29.6-24.1 & $3.3-3.8$ & $2.6-4.6$ & $1.8-0.8$ & $18.6-35.6$ & & $1.3-0-4$ & $0.1-0.1$ \\
\hline $\begin{array}{l}\text { Precipitación del trimestre más } \\
\text { húmedo }\end{array}$ & $5.4-0$ & $5.7-10-9$ & & $16.6-13.7$ & & & & \\
\hline $\begin{array}{l}\text { Precipitación del trimestre más } \\
\text { seco }\end{array}$ & & & $0.4-0.2$ & $1.9-0.5$ & & $7-15.7$ & $11.9-0.3$ & \\
\hline Altitud & $0.4-0.7$ & $5.4-3$ & $1.8-7.5$ & $0.7-1.1$ & $27.6-30.6$ & $15.4-8$ & $0.6-3.7$ & $26.2-29.8$ \\
\hline Índice de Vegetación Mejorado & $3.1-1.5$ & & & $9.4-2.5$ & & $2.2-9.6$ & $0.2-0.9$ & \\
\hline $\begin{array}{l}\text { Índice de Vegetación de } \\
\text { Diferencia Normalizada }\end{array}$ & & $5.2-7.2$ & $1.1-0.3$ & $0.5-1$ & & & $9.5-8.1$ & \\
\hline Índice de Huella Humana & $17.6-4.5$ & $41.8-26.2$ & $15-21.1$ & $9.5-12.9$ & $20.4-6.2$ & $6.5-3.2$ & $11.7-5.9$ & $0.7-0.3$ \\
\hline Total & 100 & 100 & 100 & 99.9 & 100 & 100 & 100 & 99.9 \\
\hline $\begin{array}{l}\text { \% explicado por las dos variables } \\
\text { más importantes }\end{array}$ & 52 & 70.4 & 57.5 & 37.2 & 53 & 52.9 & 58.7 & 56.3 \\
\hline $\begin{array}{l}\% \text { explicado por variables } \\
\text { superiores al } 10 \%(\mathrm{n})\end{array}$ & $83.9(4)$ & $70.4(2)$ & $91.9(5)$ & $66.6(4)$ & $90(4)$ & $76.9(4)$ & $81(4)$ & $73.1(4)$ \\
\hline AUCTest & $\begin{array}{c}0.835 \\
\pm 0.093\end{array}$ & $\begin{array}{c}0.752 \\
\pm 0.107\end{array}$ & $\begin{array}{c}0.818 \\
\pm 0.066\end{array}$ & $\begin{array}{c}0.645 \\
\pm 0.177\end{array}$ & $\begin{array}{c}0.853 \\
\pm 0.108\end{array}$ & $\begin{array}{c}0.697 \\
\pm 0.121\end{array}$ & $\begin{array}{c}0.865 \\
\pm 0.072\end{array}$ & $\begin{array}{c}0.931 \\
\pm 0.090\end{array}$ \\
\hline
\end{tabular}

geoffroyi, Leopardus colocolo, Puma concolor, Galictis cuja, Lyncodon patagonicus, Conepatus chinga, Lycalopex gymnocercus, Lycalopex culpaeus, Chaetophractus villosus, Zaedyus pichiy y Lama guanicoe) (Figuras 2 y 3). En cambio, otro grupo más pequeño presenta una distribución natural más acotada y, por lo tanto, sólo se encuentra en una parte de esta zona (Pudu puda, Hippocamelus bisulcus, Leopardus guigna y Dolichotis patagonum (Figura 1).

Aunque la mayor parte de los modelos resulta de la interacción entre diversas variables climáticas y topográficas, en la mayoría de las especies, la estacionalidad de la temperatura fue la variable más importante, con valores que van desde $0.3 \%$ para L. guigna a $46.8 \%$ para L. guanicoe (Tabla 2 y Tabla 2 bis). Además, la estacionalidad de la precipitación también fue una variable representativa en nuestros modelos, variando desde $0.1 \%$ para D. patagonum a $29.6 \%$ para L. patagonicus (Tabla 2 y Tabla 2 bis). Esto puede deberse a la marcada estacionalidad que presenta la región de la Patagonia argentina, tanto a nivel de temperatura como de precipitación. Factores locales como la topografía y el viento afectan la temperatura del aire, lo que produce una 

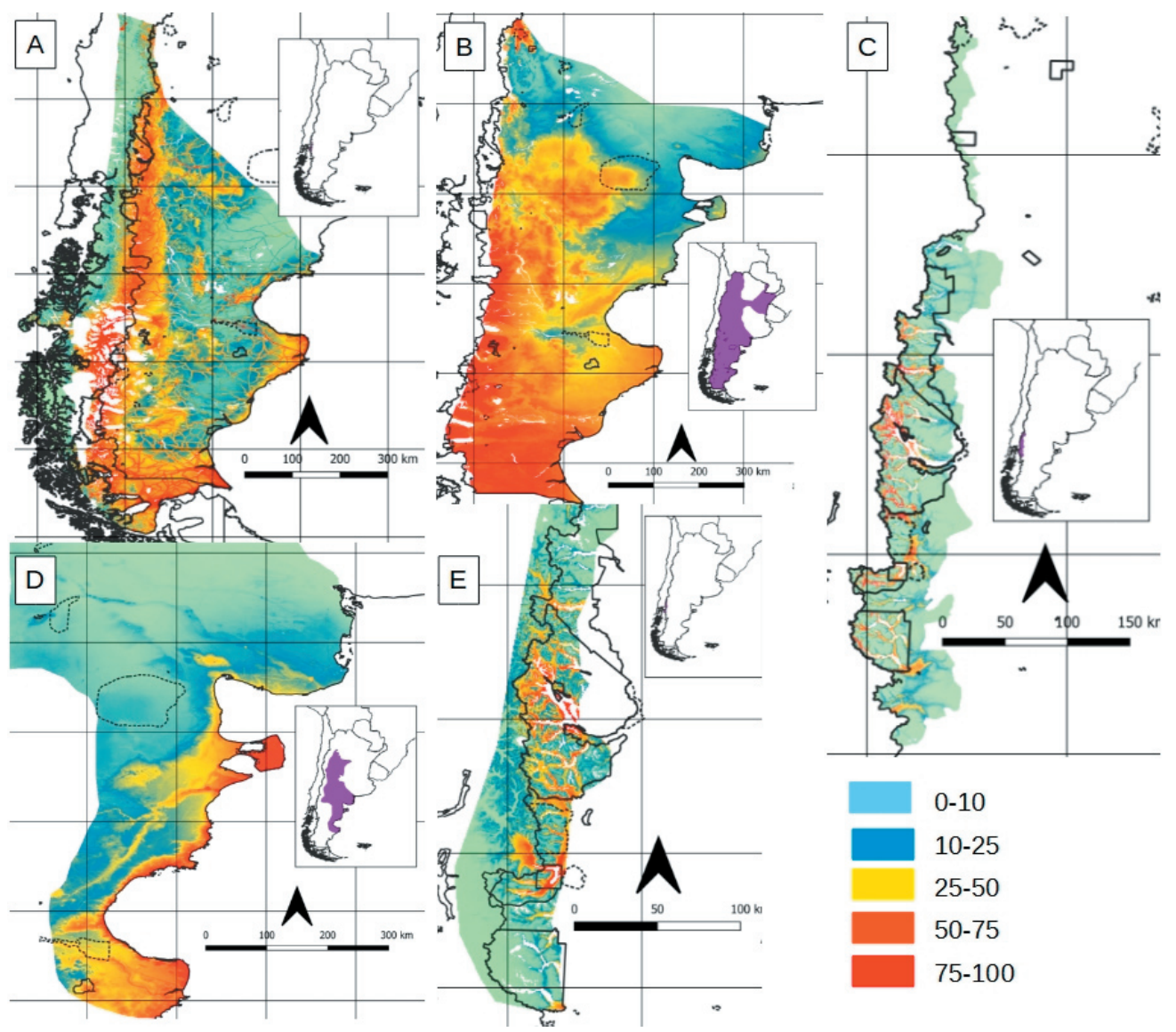

Figura 1. Modelos de distribución potencial generados para A) Hippocamelus bisulcus, B) Leopardus colocolo, C) Leopardus guigna, D) Dolichotis patagonum y E) Pudu puda. Las líneas negras representan las áreas protegidas en las categorías I y II, y las líneas punteadas representan las áreas protegidas en las categorías III a VI, según IUCN (2000).

Figure 1. Potential distribution models generated for A) Hippocamelus bisulcus, B) Leopardus colocolo, C) Leopardus guigna, D) Dolichotis patagonum and E) Pudu puda. Black lines represent protected areas in the categories I and II, and dotted lines represent protected areas in the categories III to VI, sensu IUCN (2000).

sensación térmica menor en toda la región e, incluso, más pronunciada en verano (Coronato 1993). En toda la región, las precipitaciones se concentran principalmente en invierno (Paruelo et al. 1998), ya sea en forma de nieve o de agua.

\section{Análisis de conservación}

Sólo un pequeño porcentaje de la distribución potencial de las especies se encuentra contenido dentro del sistema de áreas protegidas nacionales y provinciales de la Patagonia. En la actualidad, sólo una de las especies en estudio está en peligro de extinción: $H$. bisulcus, un ciervo nativo de la Patagonia chilena y argentina, con un $30 \%$ de la distribución potencial en las áreas protegidas categorías IUCN- I y II (APCIyII) y un $10 \%$ en las áreas categorías IUCN- III a VI (APCIIIaVI). El resto de la distribución se encuentra fuera de las áreas protegidas (AP).
Cuatro especies se encuentran en la categoría vulnerable, según categoría nacional de conservación (2019); P. puda, L. guigna, L. colocolo y D. patagonum. En el caso de P. puda, un $50 \%$ de su distribución está contenido dentro de APCIyII y un $20 \%$ en APCIIIaVI; esto puede deberse a que la distribución histórica y la actual de esta especie coincide con una red de área protegidas en el noroeste de la Patagonia (Figuras 1 y 2). La mayoría de la distribución potencial $(60 \%)$ de L. guigna y de P. puda se encuentra dentro de APCIyII, y sus distribuciones naturales coinciden casi en su totalidad con una red de AP. Para L. colocolo, la mayoría de la distribución potencial se ubica afuera de las AP (80\%). Dolichotis patagonum presenta un área de distribución menor en el este de la Patagonia, un $10 \%$ de la distribución potencial coincide con APCIyII, un 30\% APCIIIaVI y el resto se encuentra fuera de estas (Figuras 1 y 2; Tabla 3). La especie Z. pichiy está casi amenazada (i.e., fue evaluada y no satisface, actu- 

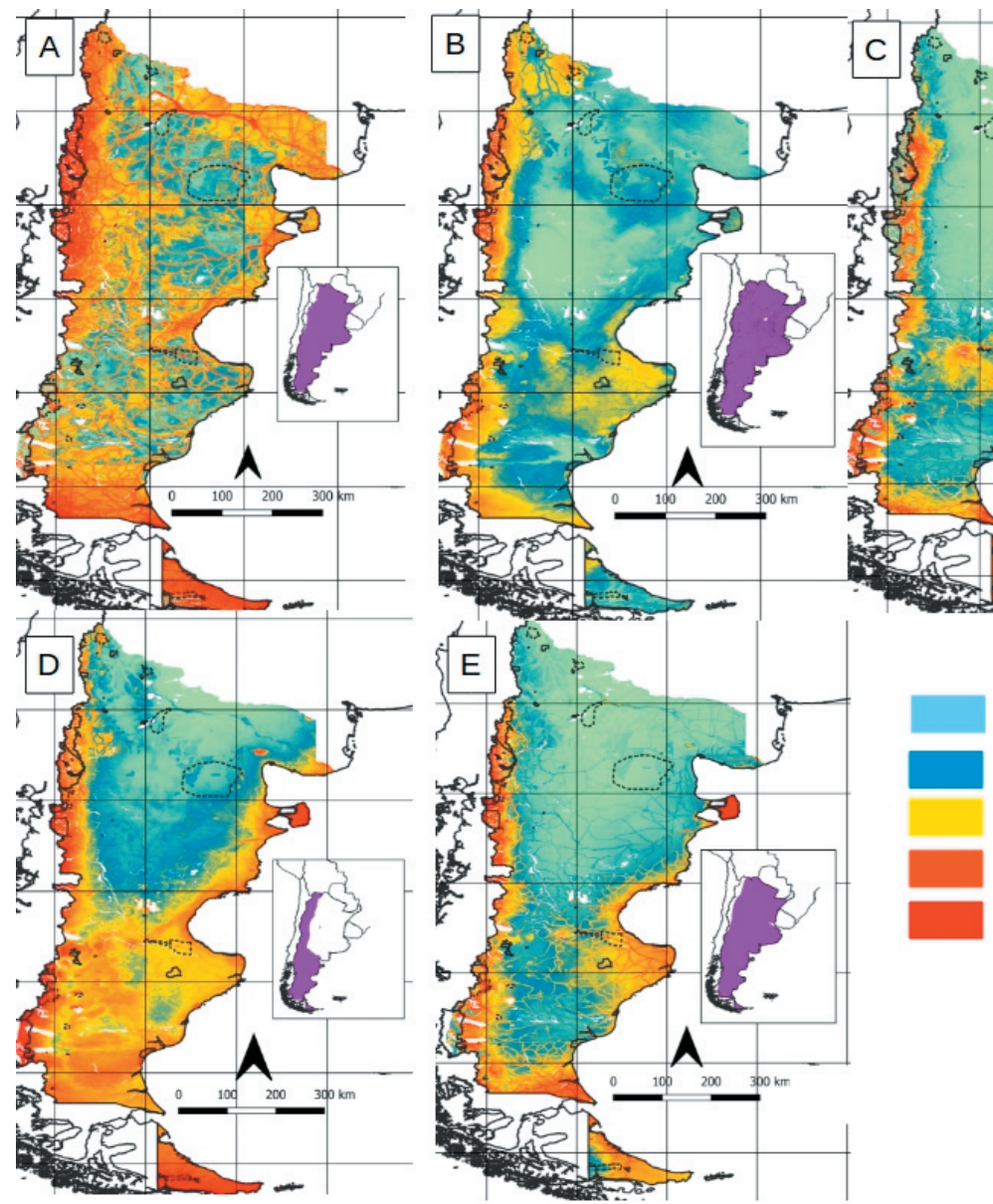

$0-10$

10-25

25-50

50-75

75-100

Figura 2. Modelos de distribución potencial generados para A) Leopardus geoffroyi, B) Puma concolor, C) Lama guanicoe, D) Lycalopex culpaeus y E) Lycalopex gymnocercus. Las líneas negras representan las áreas protegidas en las categorías I y II, y las líneas punteadas representan las áreas protegidas en las categorías III a VI, según IUCN (2000).

Figure 2. Potential distribution models generated for A) Leopardus geoffroyi, B) Puma concolor, C) Lama guanicoe, D) Lycalopex culpaeus and E) Lycalopex gymnocercus. Black lines represent protected areas in the categories I and II, and dotted lines represent protected areas in the categories III to VI, sensu IUCN (2000).

almente, los criterios para En Peligro Crítico, En Peligro o Vulnerable, pero está próxima a satisfacer los criterios, o posiblemente los satisfaga, en un futuro cercano) (IUCN 2017), y la mayor parte de su distribución potencial se ubica fuera de AP. El resto de las especies se encuentra en la categoría de Preocupación menor (i.e., fue evaluado, no cumple ninguno de los criterios que definen las categorías) (IUCN 2017), presentando una distribución potencial que se encuentra en su mayoría fuera de las áreas protegidas (superior a $60 \%$ ) (Figuras 2 y 3; Tabla 3). La figura 4 muestra que gran porcentaje de la superficie de la Patagonia es apta climáticamente y a nivel topográfico para que las especies que se encuentran dentro de las categorías en peligro de extinción y vulnerable puedan habitar.

\section{Discusión}

La evidencia de que casi toda la superficie de la Patagonia es climáticamente apta para las especies estudiadas, inclusive para las más amenazadas, como ocurre en ambientes similares en Chile (Muñoz-Pedreros and Yáñez 2009) nos invita a pensar que las causas de la retracción distribucional no están vinculadas con limitaciones impuestas por la envoltura climática, sino con otros factores derivados de la combinación de características de la biología de las especies y de la creciente presión antrópica sobre el uso de la tierra. En grandes extensiones de la Patagonia podemos encontrar un alto nivel de perturbaciones en el ambiente. En especial, esto se debe a la producción ganadera, la agricultura, la 


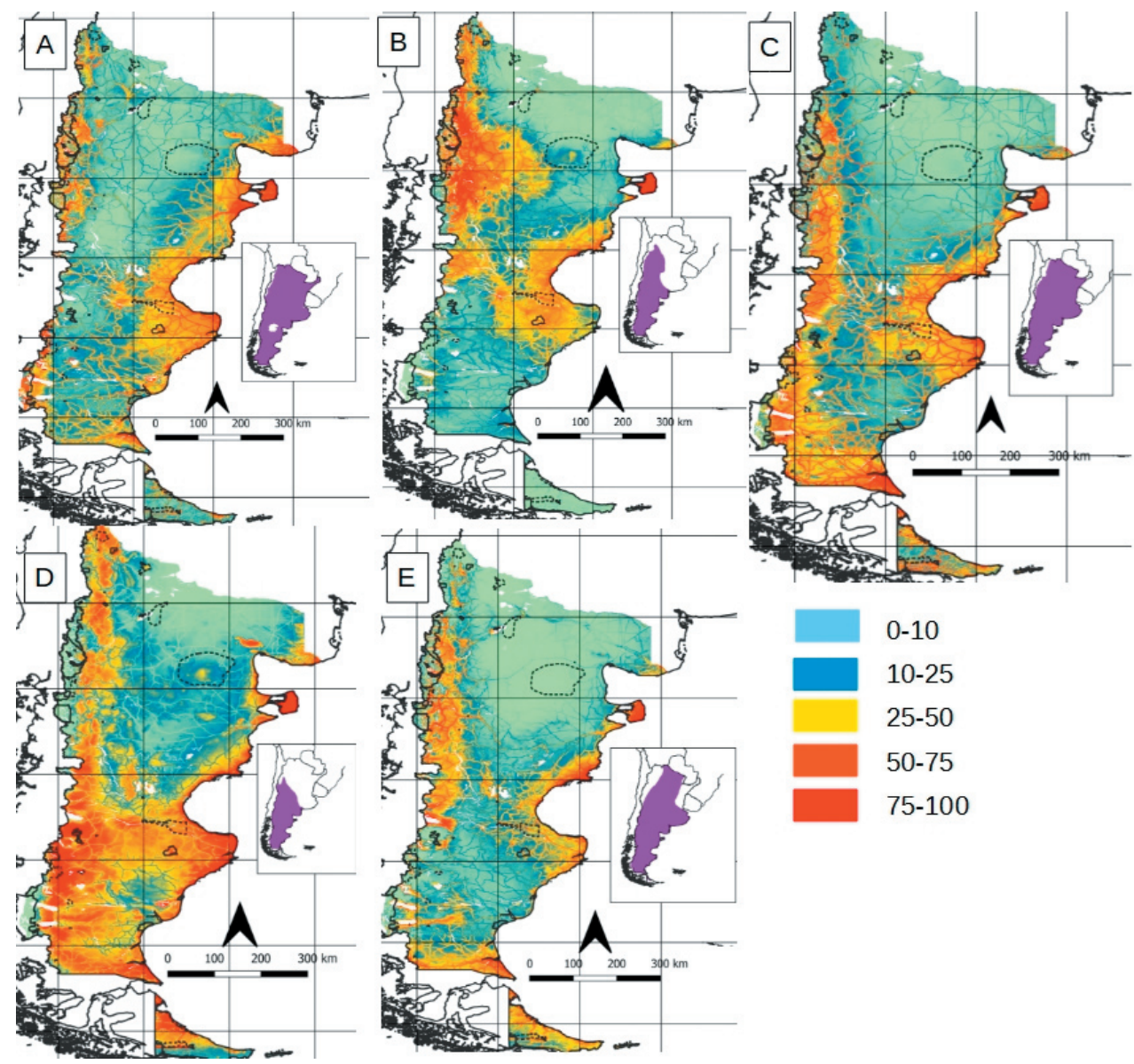

Figura 3. Modelos de distribución potencial generados para A) Galictis cuja, B) Lyncodon patagonicus, C) Conepatus chinga, D) Zaedyus pichiy y E) Chaetophractus villosus. Las líneas negras representan las áreas protegidas en las categorías I y II, y las líneas punteadas representan las áreas protegidas en las categorías III a VI, según IUCN (2000).

Figure 3. Potential distribution models generated for A) Galictis cuja, B) Lyncodon patagonicus, C) Conepatus chinga, D) Zaedyus pichiy and E) Chaetophractus villosus. Black lines represent protected areas in the categories I and II, and dotted lines represent protected areas in the categories III to VI, sensu IUCN (2000).

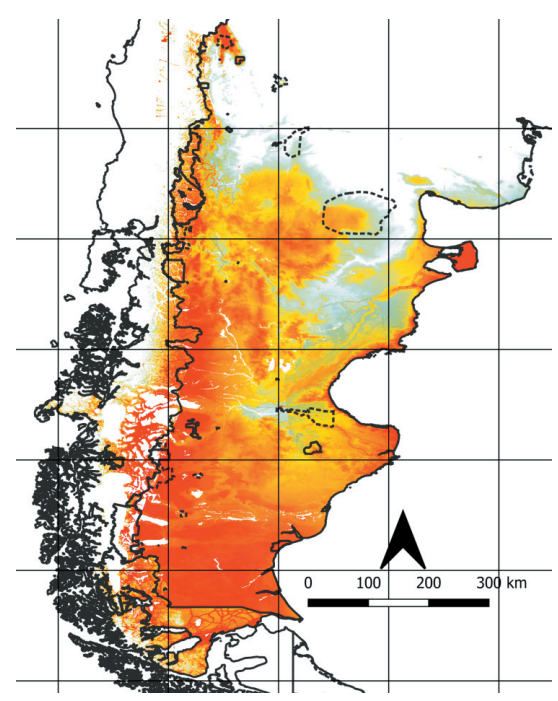

Figura 4. Modelos de distribución potencial generados para las especies que se encuentran dentro de la categorías 'peligro de extinción' y 'vulnerable' (Hippocamelus bisulcus, Pudu puda, Leopardus colocolo, Dolichotis patagonum y Leopardus guigna). Las líneas negras representan las áreas protegidas en las categorías I y II, y las líneas punteadas representan las áreas protegidas en las categorías III a VI, según IUCN (2000).

Figure 4. Potential distribution models generated for species found within the category 'endangered' and 'vulnerable' (Hippoc amelus bisulcus, Pudu puda, Leopardus colocolo, Dolichotis patagonum and Leopardus guigna). Black lines represent protected areas in the categories I and II, and dotted lines represent protected areas in the categories III to VI, sensu IUCN (2000). 
extracción de madera, la producción de leña, las actividades productivas y la reducción de individuos debido al hostigamiento por parte de actividades de caza. De esta forma se produce una distribución restringida de las especies nativas en todo el territorio.

A grandes rasgos, encontramos dos tamaños de las distribuciones potenciales: amplias y restringidas. Estas resultantes del modelo se pueden explicar simplemente por la mayor superficie de la estepa patagónica respecto de la de los bosques, que es donde se registran las distribuciones más amplias. Por el contrario, las especies confinadas a los ambientes cordilleranos y boscosos tienen áreas de distribución más restringidas. La presión antrópica directa o indirecta ha provocado la retracción distribucional hacia los sitios más inaccesibles. Lo más interesante de las predicciones son los bordes de las distribuciones potenciales, que es donde se reflejan de manera espacialmente explícita los limites fisiológicos de la especie y donde mejor funciona el modelo. Predecir la disposición espacial de una especie cuando esta se encuentra lejos de sus puntos críticos, es decir, en el centro del rango de variación de sus indicadores, es más difícil que predecir la presencia de la especie cuando está viviendo en el límite de sus posibilidades adaptativas, donde los indicadores están en valores extremos, contrastantes con la matriz de paisaje (Vale et al. 2015; Thuiller et al. 2014; Hernández et al. 2006). En estos casos, el indicador más importante es el que representa al elemento más escaso. No importa cuán complejo sea el sistema de interacciones entre la fisiología y el ambiente, el sistema colapsará cuando el elemento más escaso sea insuficiente. Las distribuciones son, entonces, amplias y compactas, y aparentemente continuas cuando la matriz de paisaje ofrece conectividad al intercambio de individuos en todo el paisaje, siendo difícil predecir la presencia en un punto determinado. En cambio, las distribuciones son restringidas y se vuelven fragmentadas cuando la matriz de paisaje ofrece resistencia a la dispersión, siendo escasos los sitios adecuados a la fisiología de la especie, pero predecibles por el modelo. Además, estas especies que presentan naturalmente un rango estrecho serán más vulnerables a los cambios de hábitat que ocurran en su distribución a causa del cambio climático.

La estacionalidad fue el factor climático de mayor potencia explicativa en nuestros modelos, tanto para la precipitación como para la temperatura. Eso se puede deber a que el clima de la Patagonia, como en casi cualquier sitio de latitud elevada, está caracterizado por una fuerte variación cíclica, tanto de las temperaturas como de las precipitaciones. La época seca coincide con las temperaturas más elevadas del verano y la época de lluvias coincide con las frías temperaturas invernales. En el centro de la región es donde se observan rangos térmicos más elevados entre el verano y el invierno y entre el día y la noche. Es plausible pensar que las especies respondan de manera diferente a los rangos estacionales. Puede hipotetizarse que, para las especies del bosque, el factor limitante tal vez esté vinculado a la economía de la humedad, escasa en las sequías del verano. En cambio, para las especies de estepa, el factor limitante puede estar vinculado a la temperatura.

Existen múltiples estudios en todo el mundo que demuestran que las áreas protegidas son necesarias, pero insuficientes, para la conservación de las especies, sobre todo para satisfacer las demandas territoriales de los grandes vertebrados (Chauvenet and Barnes 2016; Rapoport 1982). Por lo tanto, las áreas protegidas no son suficientes para disminuir el riesgo de extinción. Aunque la mayoría de las áreas protegidas de la Patagonia son regiones adecuadas para que las especies en estudio habiten, se requiere también que tengan protección fuera de estas áreas. Buscar la conectividad entre zonas y evitar la fragmentación de los ambientes naturales se garantizaría no sólo conservar las especies sino también mantener los procesos ecológicos (Sepúlveda et al. 1997).

Necesitamos realizar acciones de manejo y esfuerzos para conservar la biodiversidad y sus procesos dentro y fuera de las áreas protegidas, poniendo mayor atención a las especies que se encuentran dentro de las categorías más alarmantes como, por ejemplo, el ciervo nativo de la Patagonia, el huemul ( $H$. bisulcus). Esta especie se encuentra en la categoría en peligro de extinción debido a factores como la pérdida y degradación del hábitat, la caza furtiva, la interferencia con ungulados exóticos y la depredación por perros (Corti et al. 2010; Díaz and J. Smith-Flueck 2001). Esto conlleva a que las poblaciones sean pequeñas y se encuentren fragmentadas y confinadas a sitios de mala calidad (Flueck et al., datos no publicados; Flueck and SmithFlueck 2006; Corti et al. 2011). Nuestro modelo muestra que toda la cordillera de la Patagonia es un hábitat climáticamente apto para que la 
especie pueda sobrevivir (Rosas et al. 2017; Quevedo et al. 2017); esto se ve reflejado en la distribución actual de esta especie. Por otro lado, también muestra zonas al sur, al centro y al este de la provincia de Santa Cruz y sobre la costa este de Chubut. Esta distribución potencial está relacionada con la distribución histórica de la especie. Existen registros de que habitaba en la estepa (Vila et al. 2010), lo que indica una preferencia natural por las áreas de ecotono entre bosques y terrenos abiertos (Rosas et al. 2017; Vila et al. 2010; Díaz et al. 2013), donde existen recursos de forraje y agua en abundancia. Aunque en la actualidad la mayoría de las poblaciones se encuentran dentro de áreas protegidas, para aumentar la protección de la especie es necesario realizar acciones de manejo y protección de la especie, y promover estrategias de manejo innovadoras en las zonas con hábitats de alto valor (SmithFlueck et al. 2011).

Por su parte, el pudú (P. puda) se encuentra en la categoría vulnerable. Esta especie se ve afectada por la fragmentación, la pérdida y los cambios en el uso de la tierra, las forestaciones con plantas exóticas, la caza ilegal, la competencia y las enfermedades transmitidas por el ganado, a lo que se suma la principal causa de retroceso poblacional: la depredación por parte de perros salvajes o domésticos (Jiménez 2010; Bello 2003; Eldridge et al. 1987; Hershkovitz 1982). Esto provoca que la población disminuya y que su rango de distribución se haya reducido y fragmentado de manera considerable (Jiménez 2010; Eldridge et al. 1987; Hershkovitz 1982). Nuestro modelo muestra muy pocas zonas que presentan condiciones ambientales aptas para esta especie; en su mayoría coinciden con los parques nacionales Nahuel Huapi y Lago Puelo.

El gato huiña (L. guigna) es una de las dos especies de felinos silvestres más amenazados de América del Sur (Napolitano et al. 2012). Se encuentra en la categoría vulnerable debido a diferentes factores antrópicos como los cambios en el ambiente, la presencia de ganado y de herbívoros exóticos, el uso maderero, las plantaciones de coníferas exóticas, los incendios, la construcción de rutas, la presión inmobiliaria y el incremento de actividad turística (Monteverde et al. 2019). Estos factores provocaron una reducción en el tamaño poblacional y una disminución en el área de ocupación. Nuestro modelo mostró muy pocas zonas climáticamente ideales para esta especie, lo que puede deberse al bajo número de registros de avistajes con los cuales se trabajó. En el caso de Vale et al. (2015), estimaron un área climáticamente adecuada $\left(356000 \mathrm{~km}^{2}\right)$ mucho mayor que la actual $\left(177000 \mathrm{~km}^{2}\right)$ (Acosta and Lucherini 2008).

Aunque el gato de los pajonales (L. colocolo) posee una amplia distribución dentro de la Argentina, se encuentra expuesto a factores como la pérdida, la fragmentación y la alteración de los hábitats naturales, la frontera agropecuaria, las actividades extractivas, el atropellamiento y el envenenamiento (Lucherini et al. 2019), lo que ha provocado la disminución de su población, encontrándose en la actualidad dentro de la categoría vulnerable. El modelo muestra una amplia distribución potencial dentro de la Patagonia; esto se puede deber a que las especies con grandes rangos geográficos deberían ser más tolerantes a un espectro más amplio de condiciones climáticas, pero, entonces, su distribución no puede ser determinada únicamente por el clima (Vale et al. 2015). Por esta razón se necesita más información sobre la distribución y biología de esta especie para estimar mejor su distribución potencial.

No existe mucha información sobre la situación actual de la mara (D. patagonum). Se cree que hubo una disminución de su población, pero son necesarios estudios sobre su abundancia. En la actualidad, está categorizada como especie vulnerable, siendo amenazados sus individuos por la pérdida o la degradación del hábitat, la caza y las especies exóticas (Alonso Roldán et al. 2019). El modelo muestra a la Península Valdés como la zona más propicia para que esta especie sobreviva; coincide con su hábitat óptimo que es la estepa y matorral arbustivo.

Agradecimientos. Agradecemos a Trinidad Ruiz Barlett y Baltazar González Chávez por el apoyo y consultas sobre los modelos. A Mauro Elías por el intercambio de ideas. A los revisores anónimos de este trabajo, cuyas sugerencias aumentaron la calidad de nuestro trabajo en comparación con la primera versión. A Fundación Bariloche por su ambiente de trabajo creativo, cordial y multidisciplinario. Este trabajo ha contado con el financiamiento del PICT 2014-1558 de la ANCyT y del World Conservation Monitoring Centre (proyecto Nature Map). 


\section{REFERENCIAS}

Abramson, G., M. F. Laguna, M. N. Kuperman, J. A. Monjeau, and J. L. Lanata. 2017. On the roles of hunting and habitat size on the extinction of megafauna. Quaternary International 431:194-204. https://doi.org/10.1016/ j.quaint.2015.08.043.

Acosta, G., and M. Lucherini. 2008. Leopardus guigna. The IUCN Red List of Threatened Species. Version 2014.2. http: //www.iucnredlist.org/details/15311/0.

Alonso Roldán, V., D. E. Udrizar Sauthier, S. Giannoni, and C. M. Campos. 2019. Dolichotis patagonum. In SAyDS-SAREM (eds.). Categorización 2019 de los mamíferos de Argentina según su riesgo de extinción. Lista Roja de los mamíferos de Argentina. Versión digital: URL: cma.sarem.org.an

Astorga, F., L. E. Escobar, D. Poo-Muñoz, J. Escobar-Dodero, S. Rojas-Hucks, M. Alvarado-Rybak, et al. 2018. Distributional ecology of Andes hantavirus: a macroecological approach. International Journal of Health Geographics 17(1):22. https://doi.org/10.1186/s12942-018-0142-z.

Barve, N., V. Barve, A. Jiménez-Valverde, and A. Lira-Noriega. 2011. The crucial role of the accessible area in ecological niche modeling and species distribution modeling. Ecological Modelling 222:1810-1819. https://doi.org/10.1016/ i.ecolmodel.2011.02.011.

Bello, M. A. 2003. Ecología del pudú (Pudu pudu, Molina 1782) y su valor como especie focal, en la Provincia de Valdivia, Ecorregión Valdiviana. Tesis Licenciatura. Universidad Católica de Temuco, Temuco, Chile.

Bouchet, P. J., and J. J. Meeuwig. 2015. Drifting baited stereo-videography: a novel sampling tool for surveying pelagic wildlife in offshore marine reserves. Ecosphere 6(8):137. https://doi.org/10.1890/ES14-00380.1.

Brown, J. H. 1995. Macroecology. Journal of Mammalogy 78(1):257-260. https://doi.org/10.2307/1382661.

Bruner, A. G., R. E. Gullison, R. E. Rice, and G. Fonseca. 2001. Do parks protect tropical biodiversity? Science 291:125128. https://doi.org/10.1126/science.291.5501.125.

Chauvenet, A. L. M. and M. Barnes. 2016. Expanding protected areas is not enough. Science 353:551-552. https: //doi.org/10.1126/science.aah3762

Corti, P., U. Wittmer, and M. Festa-Bianchet. 2010. Dynamics of a small population of endangered huemul deer (Hippocamelus bisulcus) in Chilean Patagonia. Journal of Mammalogy 91:690-697. https://doi.org/10.1644/09-MAMMA-047.1.

Corti, P., A. B. A. Shafer, D. W. Coltman, et al. 2011. Past bottlenecks and current population fragmentation of endangered huemul deer (Hippocamelus bisulcus): implications for preservation of genetic diversity. Conservation Genetics 12: 119-128. https://doi.org/10.1007/s10592-009-9997-7.

Díaz, N. I., and J. Smith-Flueck. 2000. The patagonian huemul. A mysterious deer on the brink of extinction. L.O.L.A., Buenos Aires.

Díaz, P., B. I. Marqués, and A. R. Vila. 2013. Seasonal habitat use and selection of the endangered huemul deer (Hippocamelus bisulcus) in Patagonian Andes. Mammalia 77:371-380. https://doi.org/10.1515/mammalia-2012-0078.

Eldridge, W. D., M. M. MacNamara, and N. V. Pacheco. 1987. Activity patterns and habitat utilization of pudus (Pudu puda) in south-central Chile. Pp. 352-370 in C. M. Wemmer (ed.). Biology and management of the Cervidae. Smithsonian Institution Press, Washington, D.C.

Elith, J., C. Graham, R. Anderson, M. Dudik, and S. Ferrier. 2006. Novel methods improve prediction of species' distributions from occurrence data. Ecography 29:129-151. https://doi.org/10.1111/j.2006.0906-7590.04596.x

Escobar, L., A. Lira-Noriega, G. Medina-Vogel, and A. T. Peterson. 2014. Potential for spread of the white-nose fungus (Pseudogymnoascus destructans) in the Americas: use of MaxEnt and NicheA to assure strict model transference. Geospatial Health 9:221-229. https://doi.org/10.4081/gh.2014.19.

Feller, W. 1967. An introduction to probability theory and its applications. Second edition. Wiley, New York. https: //doi.org/10.1080/00224065.1970.11980411.

Fick, S. E., and R. J. Hijmans. 2017. WorldClim 2: new 1km spatial resolution climate surfaces for global land areas. International Journal of Climatology 37:4302-4315. https://doi.org/10.1002/joc.5086.

Flueck, W. T., and J. MSmith-Flueck. 2006. Predicaments of endangered huemul deer. Hippocamelus bisulcus, in Argentina: a review. European Journal of Wildlife Research 52:69-80. https://doi.org/10.1007/s10344-005-0020-4.

González, B., F. Brook, and G. Martin. 2021. Distribución y conservación de las especies de Marmosini (Didelphimorphia Didelphidae) de Colombia. PREPRINT (Versión 1) disponible en Research Square. https://doi.org/10.21203/rs.3.rs$557895 / \mathrm{v} 1$

Guisan, A., and W. Thuiller. 2005. Predicting species distribution: offering more than simple habitat models. Ecology Letters 8:993-1009. https://doi.org/10.1111/J.1461-0248.2005.00792.X.

Hernández, P. A., C. H. Graham, L. L. Master, and D. L. Albert. 2006. The effect of sample size and species characteristics on performance of different species distribution modeling methods. Ecography 29:773-785. https://doi.org/10.1111/ j.0906-7590.2006.04700.x.

Hershkovitz, P. 1982. Neotropical deer (Cervidae). Part I. Pudus, genus Pudu Gray. Fieldiana Zoology, New Series, Chicago. https://doi.org/10.5962/bhl.title.5080.

Hijmans, R. J., S. E. Cameron, J. L. Parra, P. G. Jones, and A. Jarvis. 2005. Very high resolution interpolated climate surfaces for global land areas. International Journal of Climatology 25:1965-1978. https://doi.org/10.1002/joc.1276.

International Union for Conservation of Nature. 2017. Guidelines for Using the IUCN Red List Categories and Criteria (V.13). URL: tinyurl.com/ccf62c45. 
Jiménez, J. E. 2010. Southern Pudu Pudu puda (Molina 1782). Pp. 140-150 in S. González and J. Barbanti (eds.). Neotropical cervidology: biology and medicine of Latin American deer. Funep/IUCN, Jaboticabal, Brazil.

Johnson, C. J., and M. Gillingham. 2005. An evaluation of mapped species distribution models used for conservation planning. Environmental Conservation 32:117-128. https://doi.org/10.1017/S0376892905002171.

Kearney, M., and W. P. Porter. 2004. Mapping the fundamental niche: physiology, climate, and the distribution of a nocturnal lizard. Ecology 85:3119-3131. https://doi.org/10.1890/03-0820.

Kuemmerle, T., V. C. Radeloff, K. Perzanowski, P. Kozlo, T. Sipko, P. Khoyetskyy, A. Bashta, E. Chikurova, I. Parnikoza, L. Baskin, P. Angelstam, and D. M. Waller. 2011. Predicting potential European bison habitat across its former range. Ecological Applications 21:830-843. https://doi.org/10.1890/10-0073.1.

León, R. J. C., D. Bran, M. Collantes, J. M. Paruelo, and A. Soriano. 1998. Grandes unidades de vegetación de la Patagonia extra andina. Ecología Austral 8:125-144. URL: tinyurl.com/4yhud7rz.

Lim, B. K., A. T. Peterson, and M. D. Engstrom. 2002. Robustness of ecologicalniche modelling algorithms for mammals in Guyana. Biodiversity Conservation 11:1237-1246. https://doi.org/10.1023/A:1016038501986.

Lucherini, M., E. Cuyckens, M. Morales, and J. Reppucci. 2019. Leopardus colocolo. In SAyDS-SAREM (eds.). Categorización 2019 de los mamíferos de Argentina según su riesgo de extinción. Lista Roja de los mamíferos de Argentina. https: //doi.org/10.31687/SaremLR.19.142.

Martin, G. M. 2010. Geographic distribution and historical occurrence of Dromiciops gliroides Thomas (Metatheria: Microbiotheria). Journal of Mammalogy 91:1025-1035. https://doi.org/10.1644/09-MAMM-A-347.1.

Merow, C., M. J. Smith, and J. A. Silander. 2013. A practical guide to MaxEnt for modeling species' distributions: what it does, and why inputs and settings matter. Ecography 36:1058-1069. https://doi.org/10.1111/j.16000587.2013.07872.x

Miller._. 2010__Species distribution modeling. Geography Compass 4:490-509. https://doi.org/10.1111/j.17498198.2010.00351.x

Monjeau, J. A. 2010. Conservation crossroads and the role of hierarchy in the decision-making process. Natureza and Conservação 8:1-8. https://doi.org/10.4322/NATCON.00802002.

Monjeau, J. A., E. C. Birney, L. Ghermandi, R. S. Sikes, L. Margutti, and C. J. Phillips. 1998. Plants, small mammals, and the hierarchical landscape classifications in Patagonia. Landscape Ecology 13:285-306. https://doi.org/10.1023/A: 1008012613305.

Monjeau, J. A, B. Araujo, G. Abramson, M. Kuperman, M. F. Laguna, and J. L. Lanata. 2017. The controversy space on Ouaternary megafaunal extinctions. Quaternary International 431:194-204. https://doi.org/10.1016/ .quaint.2015.10.022

Monteverde, M., M. M. Morales, E. Cuyckens, and M. Lucherini. 2019. Leopardus guigna. In SAyDS-SAREM (eds.). Categorización 2019 de los mamíferos de Argentina según su riesgo de extinción. Lista Roja de los mamíferos de Argentina. https://doi.org/10.31687/SaremLR.19.144.

Muñoz-Pedreros, A., and J. Yáñez (eds.). 2009. Mamíferos de Chile. Segunda edición. CEA Ediciones, Valdivia, Chile. http://dx.doi.org/10.4067/S0716-078X2001000300021.

Muscarella, R., P. J. Galante, M. Soley-Guardia, R. A. Boria, J. M. Kass, M. Uriarte, and R. P. Anderson. 2014. ENM eval: An R package for conducting spatially independent evaluations and estimating optimal model complexity for MaxEnt ecological niche models. Methods in Ecology and Evolution 5:1198-1205. https://doi.org/10.1111/2041-210X.12261.

Napolitano, C., J. Sanderson, W. Johnson, S. J. Brien, R. Hoelzel, R. A. Freer, N. Dunstone, K. Ritland, and E. Poulin. 2012. Population genetics of the felid Leopardus guigna in Southern South America: Identifying intraspecific units for conservation. Pp. 159-186 in M. Ruiz-García and J. Shostell (eds.). Molecular Population Genetics, Evolutionary Biology and Biological Conservation of Neotropical Carnivores. Nova Science Publishers, Inc. ISBN 978-60876-633-8.

Núñez Penichet, C., M. Cobos, J. Amaro, and A. Cañamero. 2016. Distribución potencial del género Omphalea (Euphorbiaceae) en Cuba: Aproximación a su distribución real [Potential distribution of the genus Omphalea (Euphorbiaceae) in Cuba: approach to its actual distribution]. Revista del Jardín Botánico Nacional 37:165-175. URL: tinyurl.com/2r2565wz.

Ortega-Huerta, M. A., and A. T. Peterson. 2005. Modelling spatial patterns of biodiversity for conservation prioritization in North-eastern Mexico. Diversity and Distributions 10:39-54. https://doi.org/10.1111/j.1472-4642.2004.00051.x.

Paruelo, J. M., A. Beltrán, E. Jobbágy, O. E. Sala, and R. A. Golluscio. 1998. The climate of Patagonia: general patterns and controls on biotic processes. Ecología Austral 8:85-101.

Pearson, R. G., and T. P. Dawson. 2003. Predicting the impacts of climate change on the distribution of species: are bioclimate envelope models useful? Global Ecology and Biogeography 12:361-371. https://doi.org/10.1046/j.1466822X.2003.00042.x

Peterson, A. T., M. Papes, and M. Eaton. 2007. Transferability and model evaluation in ecological niche modeling: a comparison of GARP and MaxEnt. Ecography 30:550-560. https://doi.org/10.1111/j.0906-7590.2007.05102.x.

Peterson, A., J. Soberón, R. Pearson, R. Anderson, E. Martínez-Meyer, M. Nakamura, and M. Araújo. 2011. Ecological Niches and Geographic Distributions (MPB-49). Princeton. Oxford. Princeton University Press. https://doi.org/ 10.1101/2020.07.06.185322.

Peterson, A. T., L. G. Ball, and K. P. Cohoon. 2002. Predicting distributions of Mexican birds using ecological niche modelling methods. Ibis 144:E27-E32. https://doi.org/10.1046/j.0019-1019.2001.00031.

Phillips, S. J., and M. Dudik. 2008. Modeling of species distributions with MaxEnt: new extensions and a comprehensive evaluation. Ecography 31:161-175. https://doi.org/10.1111/j.0906-7590.2008.5203.x 
Phillips, S. J., M. Dudýk, and R. E. Schapire. 2004. A maximum entropy approach to species distribution modeling. Pp. 655-662 in ICML'04 Proceedings of the 21st International Conference on Machine Learning. ACM Press, New York. https://doi.org/10.1145/1015330.1015412

Phillips, S. J., R. P. Anderson, and R. E. Schapire. 2006. Maximum entropy modeling of species geographic distributions. Ecological Modelling 190:231-259. https://doi.org/10.1016/j.ecolmodel.2005.03.026.

Prohaska, F. 1976. The climate of Argentina, Paraguay and Uruguay. Pp. 13-122 in W. Schwerdtfeger (ed.). Climates in Central and Southern America. World Survey of Climatology. Elsevier, Amsterdam, the Netherlands. https: //doi.org/10.1002/qj.49710343520.

Pulliam, R. H. 2000. On the relationship between niche and distribution. Ecology Letters 3:349-361. https://oi.org/ 10.1046/j.1461-0248.2000.00143.x.

Quevedo, P., A. Von Hardenberg, H. Pastore, J. Álvarez, and P. Corti. 2017. Predicting the potential distribution of the Endangered huemul deer Hippocamelus bisulcus in North Patagonia. Oryx 51:315-323. https://doi.org/10.1017/ S0030605315001106.

Radosavljevic, A., and R. P. Anderson. 2014. Making better MaxEnt models of species distributions: complexity, overfitting and evaluation. Journal of biogeography 41:629-643. https://doi.org/10.1111/jbi.12227.

Rapoport, E. H. 1982. Areography: Geographic strategies of species. Oxford [Oxfordshire]: Published on behalf of the Fundacioìn Bariloche by Pergamon Press.

Rosas, Y. M., P. L. Peri, A. Huertas Herrera, H. Pastore, and G. M. Martínez Pastur. 2017. Modeling of potential habitat suitability of Hippocamelus bisulcus: effectiveness of a protected areas network in Southern Patagonia. Ecological Processes 6:1-14. https://doi.org/10.1186/s13717-017-0096-2.

Ruiz Barlett, T., G. M. Martin, M. F. Laguna, G. Abramson, and A. Monjeau. 2019. Climatic constraints and the distribution of Patagonian mice. Journal of Mammalogy 100:1979-1991. https://doi.org/10.1093/jmammal/gyz149.

Sanderson, E. W., J. Malanding, M. A. Levy, K. H. Redford, A. V. Wannebo, and G. Woolmer. 2002. The Human Footprint and the Last of the Wild: The human footprint is a global map of human influence on the land surface, which suggests that human beings are stewards of nature, whether we like it or not. BioScience 52:891-904. https: //doi.org/10.1641/00063568(2002)052[0891:THFATL]2.0.CO;2]

Schiaffini, M. I. 2014. Ensambles de pequeños carnívoros (Carnivora: Mustelidae y Mephitidae) en Patagonia: taxonomía, distribución y repartición trófica. Tesis de Doctorado. Universidad Nacional de La Plata, La Plata, Argentina.

Schiaffini, M. I., G. M. Martin, A. L. Gimenez, and F. J. Prevosti. 2013. Distribution of Lyncodon patagonicus (Carnivora, Mustelidae): changes from the Last Glacial Maximum to the present. Journal of Mammalogy 94:339-350. https: /doi.org/10.1644/12-MAMM-A-155.1

Sepúlveda, C., A. Moreira-Muñoz, and P. Villarroel. 1997. Conservación biológica fuera de las áreas silvestres protegidas. Ambiente y Desarrollo 2:48-58. URL: bibliotecadigital.infor.cl/handle/20.500.12220/8487.

Smith-Flueck, J. M., J. Barri, N. Ferreyra, A. Núñez, N. Tomas, J. Guzman, and J. Jimenez. 2011 Advances in ecology and conservation of Hippocamelus species in South America. Animal Production Science 51:378-383. https://doi.org/ 10.1071/AN10287.

Soberón, J., and A. T. Peterson. 2005. Interpretation of models of fundamental ecological niches and species' distributional areas. Biodiversity Informatics 2:1-10. https://doi.org/10.17161/bi.v2i0.4.

Svenning, I. C. and F. Skov. 2004. Limited filling of the potential range in European tree species. Ecology Letters 7: 565-573. https://doi.org/10.1111/j.1461-0248.2004.00614.X

Thuiller, W., L. Brotons, M. B. Araujo, and S. Lavorel. 2004. Effects of restricting environmental range of data to project current and future species distributions. Ecography 27:165 172. https://doi.org/10.1111/j.0906-7590.2004.03673.x.

UICN. 2012. Categorías y Criterios de la Lista Roja de la UICN: Versión 3.1. Segunda edición. Gland, Suiza y Cambridge, Reino Unido: UICN. vi + Pp. 34. Originalmente publicado como IUCN Red List Categories and Criteria: Version 3.1. Second edition. (Gland, Switzerland and Cambridge, UK: IUCN, 2012).

Vale, M. M., M. L. Lorini, and R. Cerqueira. 2015. Neotropical Wild Cats Susceptibility to Climate Change. Oecologia Australis 19: 63-88. https://doi.org/10.4257/oeco.2015.1901.05.

Veblen, T. T., and D. C. Lorenz. 1988. Recent vegetation changes along the forest/steppe ecotone of northern Patagonia. Annals of the Association of American Geographers 78:93-111. URL: jstor.org/stable/2563442.

Vila, A. R., C. Saucedo, D. Aldridge, E. Ramilo, and P. Corti. 2010. South andean huemul Hippocamelus bisulcus (Molina 1782). Pp. 89-100 in J. M. Duarte and S. González (eds.). Neotropical cervidology: biology and medicine of Latin American deer. FUNEP-IUCN, Jaboticabal, Brazil.

Warren, D. L., and S. N. Seifert. 2011. Ecological niche modeling in MaxEnt: the importance of model complexity and the performance of model selection criteria. Ecological Applications 21:335-342. https://doi.org/10.1890/10-1171.1.

Wisz, M. S., R. J. Hijmans, J. Li, A. T. Peterson, C. H. Graham, A. Guisan, and N. P. S. Distribut. 2008. Effects of sample size on the performance of species distribution models. Diversity and Distributions 14:763-773. https://doi.org/10.1111/ j.1472-4642.2008.00482.x.

Zurell, D., J. Franklin, C. König, P. J. Bouchet, C. F. Dormann, J. Elith, G. Fandos, X. Feng, G. Guillera-Arroita, A. Guisan, J. J. Lahoz-Monfort, P. J. Leitão, D. S. Park, A. T. Peterson, G. Rapacciuolo, D. R. Schmatz, B. Schröder, J. M. Serra-Diaz, W. Thuiller, K. L. Yates, N. E. Zimmermann, and C. Merow. 2020. A standard protocol for reporting species distribution models. Ecography 43:1261-1277. https://doi.org/10.1111/ecog.04960. 